\title{
A comparative study of the mastery of fundamental movement skills between different cultures
}

\author{
Fernando Garbeloto dos Santos ${ }^{1 *}$, Matheus Maia Pacheco ${ }^{2}$, Luciano Basso ${ }^{1}$, Go Tani ${ }^{1}$
}

ORIGINAL ARTICLE

\begin{abstract}
It has been suggested that guidance, encouragement and opportunity are crucial factors for children's motor development. They are especially important to promote the mastery of fundamental movement skills. The present study investigated the association between two elements related to those factors - proposed curriculum of physical education and the most popular sport in a given country - and the incidence of mastery in the FMS in children from 4 to 10 years of age. In order to carry out a cross-cultural comparison, three studies from different countries (Portugal, China and USA) that used TGMD-II were selected. The results indicated association between proposed curriculum and the number of children mastering FMS. This association increases in older age groups of children from USA, the most structured curriculum related to FMS, presenting the highest average percentage of mastery. Additionally, we did not find association of the most popular sports in a given country with the mastery of the related skill. These results are interpreted as evidence for the importance of guidance on the mastery of FMS.
\end{abstract}

Keywords: TGMD-II, popular sport, curriculum proposal

\section{INTRODUCTION}

A Fundamental movement skills (FMS) (Wickstrom, 1977) are considered to be the basis of more advanced patterns of movements typical of daily living activities and sports (Clark \& Metcalfe, 2002; Haubenstricker \& Seefeldt, 1986). Furthermore, studies have shown a consistent relation between acquisition of these movement patterns and child participation in physical activities and sports which, in turn, would promote an active lifestyle (Barnett, Van Beurden, Morgan, Brooks, \& Beard, 2008; Okely, Booth, \& Patterson, 2001; Ridgway et al., 2009). Thus, studies seeking for the understanding of the factors that contribute to the acquisition of FMS are much needed. Seefeldt and Haubenstricker (1982) indicated that $60 \%$ of American children around 6/7 years of age mastered seven out of eight FMS. Although these skills are believed to be practiced in different cultures (Burton \& Miller, 1998), recent studies have shown that children from different countries do not reach the same level of mastery at either 7 or later ages (Goodway, Robinson, \& Crowe, 2010; Okely \& Booth, 2004; Valentini, 2012).

These differences can be linked to several factors. We underscore the lack of opportunity, encouragement, and guidance for physical activity practice (Gallahue, Ozmun, \& Goodway, 2013; Tani, 2011). With regard to guidance, physical education classes at school would play an important role (Manoel, 1994; Tani, 2011). Studies have shown that interventions with the specific goal of improvement in FMS resulted in good levels of mastery (Hardy, Barnett, Espinel, \& Okely, 2013; van Beurden et al., 2003; van Beurden, Zask, Barnett, \& Dietrich, 2002). However, these studies have introduced an external intervention program into schools, which may not reflect the content of the general physical education classes of a given region.

In general, the content taught in physical education classes follow guidelines created by

Manuscript received at March 22 $2^{\text {nd }} 2016$; Accepted at May $10^{\text {th }} 2016$

\footnotetext{
${ }^{1}$ School of Sports and Physical Education of University of São Paulo, São Paulo, Brazil

${ }^{2}$ Department of Kinesiology - The University of Georgia - Athens, United States of America.

* Corresponding author. Professor Mello Moraes 65, São Paulo, Brazil E-mail: fegarbeloto@gmail.com
} 
competent governing bodies. Portugal follow the Organization Curriculum and Programs (Departamento do Ensino Secundário, 1998)) which is fairly comprehensive, but do not specifically emphasize the development of FMS. Rather, the objectives underline aspects including socialization among students, learning sports, and popular recreational activities. In contrast, the physical education guidelines in the United States of America (USA) and China aim specifically to the development of FMS (AAHPERD Curriculum Framework Task Force, 2014; Curriculum Development Council [CDC], 2002). The American curriculum illustrates in detail the requirements of each age group in terms of mastering the FMS (e.g., in the end of pre-school, children should master the hop, gallop, run and slide). Similarly, the Chinese curriculum base its goals on the development of FMS, but share the emphasis with sports learning, cultural games and activities. Thus, provided the relation between the proposed curriculum and the actual content of physical education classes, we expect that the curriculum proposals would influence the number of children mastering the FMS and/or the speed at which this number is reached.

Despite the fact that guidelines of each country serve as a guide to plan the content that will be developed in schools around the world, there is little information about the relation between the content of national physical education proposal (e.g. Organization Curriculum and Programs in Portugal) and the motor performance in children. One of few studies that investigated these relations, carried out by Koutedakis and Bouziotas (2003), assessed aspects of health and physical activity levels (e.g. time spent in intensive physical activity) in Greek adolescents. Nevertheless, we could not find studies that relate the national curriculum contents to the quality of movement (e.g. level of mastery in FMS). A cross cultural analysis comparing different proposals would provide empirical support for future curriculum proposals. Additionally, distinct percentages of mastery from different studies cannot imply a direct inference of differences between countries - they may occur by chance. These studies often have different sample sizes for each age group and it is not reliable to infer from descriptive statistics. Direct inferential statistics are needed to support such a claim.

Beyond the school environment, the most popular sport in a given country is pointed as a potential influence to the developmental process of FMS. For example, Wong and Cheung (2006) suggest that cultural context in western countries will provide more opportunities for children practice striking, throwing and rolling skills provided these are the main popular sports of that part of the globe. This phenomenon would occur largely because children have more spaces for practice (i.e., greatest opportunity) and encouragement (i.e., encouragement of parents and friends) to perform specific sports. In addition, marketing and media would reinforce the practice of certain sports influencing the involvement of children at all ages. Sports technical structure involves specific skills and FMS. Different sports provide varying levels of engagement in certain FMS, and as a result some of these are encouraged over others.

In Portugal, soccer is the most popular sport (Marivoet, 2000). In this case, the high availability of appropriate space (e.g., soccer fields, indoor arenas) provides ample opportunity for children to practice. With a cooccurrence to a higher encouragement, we expect that children from Portugal will have more practice in the kick - a FMS that is the basis of this sport. This would result in a greater number of Portuguese children with mastery in this FMS from earlier age in comparison to children from other countries where soccer does not exert as much influence. In China the most popular sports are table tennis and basketball while in the USA there is preference for American football and baseball (Chen, 2015; Schwartz \& McGarry, 2014). Despite the use of sporting context (i.e. most popular sport) as an argument to explain possible differences in the mastery of certain FMS in children from different countries (Afonso et al., 2009; Wong \& Cheung, 2006), to date, there is no empirical evidence that support such association.

In this scenario, the present study investigated the association between proposed 
curriculum of physical education and sports culture in three different regions (Hong Kong China, 10 states in USA and Madeira Island Portugal) and the incidence of mastery in the FMS in children from 4 to 10 years of age. Based on the ideas and evidences above mentioned, this study establishes the following hypotheses:

Hypothesis 1. The proposed curriculum of physical education in each country influences child mastery in FMS. Given the specificity of USA and Chinese curriculum guidelines in relation to FMS, children from Hong Kong and USA would show higher incidence in mastery the FMS than children from Madeira Island. Still, the USA would overcome Hong Kong indices due to its well-defined goals for the development of FMS.

Hypothesis 2. The most popular sport favors the development of certain FMS. This was answered by the observation of the number of children mastering the FMS related to the sport of greatest popularity. Thus, the kicking would be the FMS with more children demonstrating mastery in Madeira Island (related to soccer), overhand throwing and the striking would take precedence in the USA (related to American football and baseball), and the stationary dribble would be more prevalent in Hong Kong (related to basketball).

\section{METHOD}

\section{Selection of studies}

In order to carry out a cross-cultural comparison, we searched for studies in different countries with the following criteria: the study assessed the performance in the FMS with a minimum sample of seventy subjects per age group - ensuring a power analysis 0.99 for a medium effect; included ages from 4 to 10 years of life; and provided similar male e female participants - ensuring an unbiased sample. The following keywords were used: (*fundamental movement skills*, *fundamental pattern of movement*, *TGMD-II*, *proficiency*, *mastery*) that might appear in the title, abstract, or keywords. In order to meet the selection criteria, each study should evaluate healthy children ages 4 to 10 who had not undergone any motor intervention program, and used the TGMD-II test to assess the performance of FMS. Research sources used were: PubMed, Google Scholar, Web of Science. Three studies, each from a different country (Portugal, China, USA), were selected.

The TGMD-II (Ulrich, 2000) assesses 12 fundamental movement skills, six locomotor movements (run, gallop, hop, leap, horizontal jump, slide) and six object control movements (striking a stationary ball, stationary dribble, catch, kick, overhand throw, underhand roll). All skills have a number of performance criteria ranging between 3 and 5 . If the child shows mastery in a particular criterion, it receives 1 (one) point, otherwise it gets 0 (zero). For each skill, the child has two trials to perform the movement pattern.

Table 1

Studies (samples) selected or the present study.

\begin{tabular}{ccccccccccc}
\hline \multirow{2}{*}{ Author (Year) } & \multirow{2}{*}{ Country / City } & 4 & 5 & 6 & 7 & 8 & 9 & 10 & \multirow{2}{*}{ Boys/Girls } & \multirow{2}{*}{ Total } \\
& & & & Age group sample (n) & & \\
\hline Wong (2002) & China/ Hong Kong & 245 & 270 & 167 & 127 & 89 & 108 & 107 & $675 / 553$ & 1228 \\
Ulrich (2000) & USA/10 States & 114 & 103 & 146 & 165 & 207 & 179 & 179 & $604 / 604$ & 1208 \\
Afonso (2009) & Portugal/Madeira Island & 95 & 107 & 113 & 103 & 102 & 104 & 167 & $426 / 427$ & 853 \\
\hline
\end{tabular}

Three studies were identified, as shown in Table 1 (Afonso et al., 2009; Ulrich, 2000; Wong \& Cheung, 2006). All studies considered mastery when the children met all criteria in both attempts.

All cited studies followed the standards set by Ulrich (2000) for the training of the experimenters and the reliability coefficients in both movement classes. All participants of the selected studies had regular classes of Physical Education in their countries, and after 6 years of age the frequency was 2 weekly classes for China and USA and 3 classes for Portugal; the classes lasted between 40 and 50 minutes. 


\section{Statistical Analyses}

The dependent variable of this study was the average percentage of children who have attained mastery in the FMS per region (cities/states/island). We performed a chisquare test to analyze whether there was an association between the percentage of children with mastery in FMS and the respective countries. The magnitude of the association was measured by Cramer's V statistic. This statistic provides values between zero and one, with one (1) representing total association between categories and zero representing none.

We calculated the odds ratio (OR) of a given country presenting children with mastery of the FMS in comparison to the other two countries. This was done to verify whether the incidence of children mastering the FMS in a given country surpasses the incidence of the other two altogether. Each country was compared in terms of the mean frequency of children with mastery per age group with the relative frequency of all the other countries summed up. The values of OR can vary from zero to any positive number. We assessed these values in terms of its confidence interval to evaluate its significance. Values significantly higher than one (1) delineates that the given country has more chances to present children with mastery than the other two countries summed up. Values significantly smaller than one show that the given country has fewer chances. Any value that does not differ from one (1) shows that the country has similar chances of presenting children mastering the given FMS.

To investigate the sporting context influence on children proficiency in a specific FMS, we compared the probability of a given country to present higher percentages of children mastering this specific FMS than all other FMS for each age group. For instance, we compared the probability of Portugal present higher percentages of children mastering kicking (given the influence of soccer) than all other FMS for each age. We compared the percentages using the z-score of the difference using a one-tailed distribution (i.e., we tested the alternative hypothesis of the influenced FMS to show higher percentages than all other FMS). All analyses were performed using Matlab 2013 and SPSS 17.0 software.

\section{RESULTS}

The chi-square test detected a significant association between performance on FMS and the evaluated regions. The association values tend to increase over time (Table 2). Figure 1 presents the mean percentage of children demonstrating mastery per age, region, and FMS. Around 4 years of age children had similar performance, however, over the years the difference between the number of children from different countries who have reached mastery tended to increase.

Table 2

Association between countries and the level of mastery in each age

\begin{tabular}{cccc}
\hline Age & $\chi^{2}(3)$ & p-value & Cramer's V \\
\hline 4 & 2.54 & .278 & .075 \\
5 & 5.0 & .70 & .103 \\
6 & 10.75 & .005 & .159 \\
7 & 9.53 & .008 & .155 \\
8 & 14.56 & .001 & .191 \\
9 & 12.40 & .005 & .178 \\
10 & 11.63 & .005 & .160 \\
\hline Note: $\chi^{2}=$ Chi-square values &
\end{tabular}

Children rate from 4 to 10 years of age with mastery in FMS: relationship with the curriculum

Figure 1 presents the average percentage of children with mastery per age.

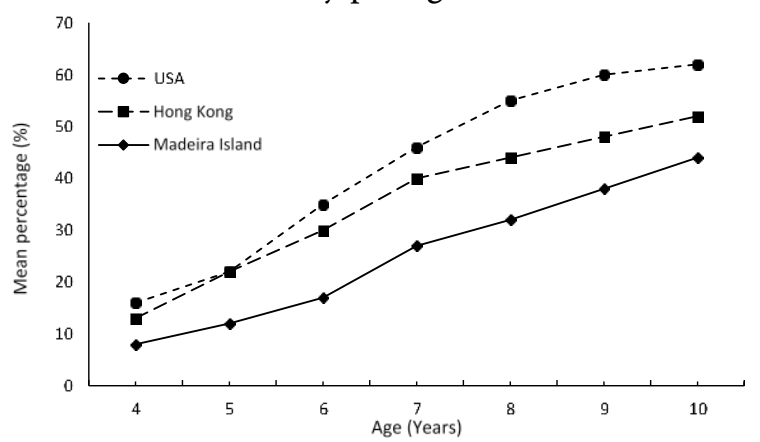

Figure 1. Mean percentage of children with mastery for all FMS per age group.

It is noteworthy that the curriculum proposal of China is directed to children older than the $6 / 7$ years old, so after this age we would find significant increments in the number of children with mastery. Children from USA presented the 
highest average percentage of children with mastery in all ages followed by children from Hong Kong - China and children from Madeira Island - Portugal respectively.

Table 3

The Odds Ratio for all FMS in each age.

\begin{tabular}{lccccccc}
\hline \multicolumn{7}{c}{ Age } \\
\hline Country & 4 & 5 & 6 & 7 & 8 & 9 & 10 \\
\hline China & 1.05 & 1.39 & 1.15 & 1.05 & 0.85 & 0.85 & 0.96 \\
USA & 1.44 & 1.17 & $1.64^{*}$ & $1.63^{*}$ & $2.02^{*}$ & $1.93^{*}$ & $1.83^{*}$ \\
Portugal & 0.56 & 0.49 & 0.42 & 0.48 & 0.44 & 0.50 & 0.55 \\
\hline Note: ${ }^{*} p<0.05$ & & & & & &
\end{tabular}

Table 3 presents the results of the odds-ratio analyses. In consonance with the descriptive results, children from US showed the highest chances to present mastery in the FMS at all ages (significant values from the six-year-old group to the ten-year-old group). Children from Hong Kong showed higher chances to present mastery while children from Madeira Island showed smaller chances. Nevertheless, the chances for children from these latter countries did not approach significant levels. Additionally, only the US presented equal or greater percentage of children with mastery in the FMS than the expected $60 \%$ - and it occurred only for older groups (9- and 10-year-old groups).

\section{Incidence of children demonstrating mastery: most popular sport}

Table 4 presents the percentage of children mastering the FMS to be influenced by the most popular sport of each country and the mean percentage of all other FMS. Observing the mean percentage of children with mastery in all FMS, we did not find any effect of most popular sports in all three regions. Table 5 presents the resultant scores- $z$ of the percentage comparison between the influenced FMS and the mean of all other. As can be observed, we did not find any effect of most popular sports in the mastery of these specific skills when compared with the others FMS. The results are maintained even when we considered only the object control skills (not shown).

Table 4

Mean percentage of children with mastery in all FMS and total percentage of children with mastery in the specific sporting culture skill.

\begin{tabular}{|c|c|c|c|c|c|c|c|c|}
\hline \multirow{2}{*}{ Country } & & \multicolumn{7}{|c|}{ Age } \\
\hline & & 4 & 5 & 6 & 7 & 8 & 9 & 10 \\
\hline \multirow{2}{*}{ China } & All FMS & 14 & 24 & 32 & 40 & 44 & 48 & 51 \\
\hline & Dribbling & 4 & 5 & 18 & 39 & 46 & 47 & 63 \\
\hline \multirow{2}{*}{ USA } & All FMS & 16 & 23 & 35 & 46 & 55 & 61 & 63 \\
\hline & Throwing & 12 & 18 & 30 & 41 & 50 & 52 & 55 \\
\hline \multirow{2}{*}{ USA } & All FMS & 16 & 23 & 36 & 47 & 56 & 61 & 63 \\
\hline & Striking & 12 & 17 & 21 & 35 & 41 & 47 & 49 \\
\hline \multirow{2}{*}{ Portugal } & All FMS & 8 & 12 & 17 & 27 & 32 & 38 & 44 \\
\hline & Kicking & 2 & 4 & 1 & 2 & 2 & 4 & 24 \\
\hline
\end{tabular}

Note: All FMS: The sum of \% of children with mastery in all FMS divided by the number of assessed skills (the specific sports culture skill was not included).

Table 5

The score-z values for the difference between the comparison with the sporting specific skill and the other 11 skills.

\begin{tabular}{cccccccc}
\hline & \multicolumn{7}{c}{ Age } \\
\hline Country (Skill) & 4 & 5 & 6 & 7 & 8 & 9 & 10 \\
\hline China (Dribble) & -2.89 & -4.78 & -2.10 & -0.03 & 0.27 & -0.06 & 1.38 \\
USA (Strike) & -0.89 & -0.94 & -2.41 & -2.15 & -3.10 & -2.69 & -2.69 \\
USA (Throw) & -0.89 & -0.75 & -0.51 & -0.90 & -1.03 & -1.64 & -1.52 \\
Portugal (Kick) & -1.90 & -2.35 & -4.63 & -5.76 & -6.62 & -7.14 & -4.37 \\
\hline
\end{tabular}

Note: the critical value for a difference was 1.645 - no comparison reached the critical value. 


\section{DISCUSSION}

This study investigated the association between most popular sport in the country and curriculum guidelines of Physical Education with the incidence of children demonstrating mastery in the FMS. To accomplish this goal, we selected three studies that described children from 4 to 10 years of age from four different regions (Madeira Island - Portugal, Hong Kong - China and 10 different states in USA). It was speculated that the specificity of the curriculum guidelines - in terms of the development of the FMS - would influence (positively) the incidence of children who achieve skill mastery. Secondly, the emphasis of each most popular sport would result in a large number of children mastering in a specific FMS. The results of this study corroborate only with the first hypothesis. Children from USA presented higher incidence than children from Hong Kong and Madeira Island on the indices of mastering the FMS in association with the observed specificity of the respective curriculum guidelines for Physical Education.

Due to influence of the most popular sport (e.g. soccer in Portugal), we expected a higher prevalence of children attaining mastery in certain skills (e.g. kick in Portugal). However, the results expressed in table 4 show that compared to other skills, the sport-related FMS did not demonstrate higher prevalence of mastery. For example, children from Madeira Island were less likely to present mastery in kicking at almost every age. In this region, kicking appears to be one of the skills with the lowest number of children mastering it. Although we observed that children from Hong Kong and USA showed a larger incidence of mastery in their respective skills, the table 5 shows that these relations were not significant for any country.

Based on these results, one can deduce that most popular sport does not lead to higher rates of children mastering skills underscored by the most popular national pastimes. Our expectation was based on the opportunities and encouragement that these sports would provide for the practice of certain skills. Thus, these factors, isolated, are not sufficient to promote mastery of FMS. Our results corroborate with those of Wang (2004) and Rimmer and Kelly (1989). These studies compared children playing freely in a structured space (i.e., a space with specific structure to support practice of various skills) with children performing activity under the guidance of a physical education professional. The results showed that those who received guidance were the only ones that showed significant improvements in the performance of FMS. In other words, the addition of proper guidance is necessary in conjunction with opportunity and incentive.

Nevertheless, these results should be interpreted with caution. There is an assumption that girls would be less encouraged to participate in games, sports and physical activities geared primarily to males (e.g., soccer in Portugal, American football in the USA) (Barnett, van Beurden, Morgan, Brooks, \& Beard, 2010; Butterfield, Angell, \& Mason, 2012; Goodway et al., 2010; Thomas \& French, 1985; Valentini, 2012). Moreover, "male-related" sports are categorized differently in depending on the culture. Soccer, for instance, is predominantly practiced by men in Portugal, but is one of the dominant athletic activities performed by girls in the United States. Despite this potential influence on our results, some studies show that this might not be the case. Afonso et al. (2009) showed that the best skill in Madeira Island considering girls and boys separately - does not relate to the most popular sport. Although Ulrich (2000) and Wong and Cheung (2006) have not evaluated the prevalence of mastery by gender, the results found by Pang and Fong (2009) in Hong Kong and Butterfield, Angell, and Mason (2012) in the United States showed that sex also did not influence the prevalence of mastery. Based on these results, it is possible to argue that sex was not a factor that influenced the prevalence of mastery in the skill associated with the most popular sport.

In regard to the curriculum guidelines goals for Physical Education, Table 2 shows that the mastery of the FMS is strongly related to the specificity of each program. Consequentially, the results expressed in Table 3 demonstrated that children from Madeira Island - in which the curriculum has general (unspecific) goals - had 
less chances to present mastery in the FMS when compared to children from Hong Kong that has more directed goals in its guidelines. The USA (country with the most direct and specific goals toward the development of FMS) had the greatest chance to present mastery in various skills.

Accordingly, studies showed that goal directed approaches for developing FMS result in significant improvements in performance (Jones et al., 2011; Logan, Robinson, Wilson, \& Lucas, 2012; McKenzie, Alcaraz, Sallis, \& Faucette, 1998). Two Australian projects serve as examples: "Move it groove it" and "Get skilled get active". These projects had as the specific goal to increase the number of children with mastery in FMS. Their findings showed that after the project began, there were significant increases in the number of children who performed the FMS proficiently (Hardy et al., 2013a; van Beurden et al., 2003, 2002). In this case, specific goals probably provide more opportunity for practice and proper guidance, which leads to better performances in FMS.

Besides the specific proposal of developing FMS in USA, another important aspect to be taken into consideration in association with the superior results of North American children may be the cultural bias of the test. Given that TGMDII is an North American test, the skills assessed were based on the skills proposed by the National Association for Sport and Physical Education (2004). Thus, it can be said that the development of such skills may be part of the US cultural context, which caused an elevated number of children demonstrating mastery in FMS.

Our analysis allows us to make an extra consideration about the percentage of children who demonstrated mastery over the years. Results found by Seefeldt and Haubenstricker (1982) showed that at 7 years of age, $60 \%$ of children demonstrated mastery of 7 of 8 tested FMS (throwing, kicking, running, jumping, catching, dribbling, hopping, and skipping). This is contrary to our findings. When we consider the number of children with mastery in a specific skill, it appears that it is rare to observe numbers greater than $60 \%$ in the mastery, even when looking at age groups ranging from 7 to 10 years. Considering the mean percentage of children with mastery in all FMS, only age groups equal or older than the 9-year-old group from the US showed values equal or greater than $60 \%$ (Table 4). In this way, is necessary to reconsider the expectancy of the moment at which children will show mastery in these skills - it can be said that $60 \%$ of mastery at 7 years of life is not the norm.

Another consideration is about the different trajectories in performance. When comparing the prevalence of mastery between children from USA, Hong Kong and Madeira Island, one can argue that there is no equifinality effect (i.e., to achieve the same end point starting from different initial points) in the development of FMS (Figure 1). That is, there is no point of convergence even at the 10-year-old group. Based on this result, it can be speculated that only the maturational process (which occurs due to aging) does not promote similar levels of motor performance. The differences between countries in older groups can be inferred from the specificity of the curriculum structure. The higher frequency of North American children with mastery can be linked to two factors: first, the same FMS is focused in different school grades; second, the curriculum specifies how many components of FMS children must perform well in each grade. For instance, we can mention the proposed guideline for the overhand throw: in grade 2 children must show 2 of 5 components being performed in the mature form, in grade 3, 3 of 5 components, and in grade 4 children must perform the overhand throw in the mature form. These two factors give support to the relevance of an increased practice of the same FMS over the years, and provide guidelines for the physical education teachers in school settings. When we consider a country that does not provide such a structure or requirements (e.g., Portugal), the expectation of change per grade (or year) decreases as the results of this study show.

Our results point out that it is necessary specific proposals to the development of FMS. Tani (2011) suggests that without the influence of external factors (proper guidance, encouragement and opportunity) children will have difficulty mastering the FMS. This is of great concern as there is significant interdependence between the stages of motor development. Low 
performance in the FMS can create a barrier for later stages of development (Seefeldt, 1980) that result in a detrimental reduction of physically active children (van Beurden et al., 2002). Our study investigated the aforementioned factors indirectly, but our findings support this relation.

The influence of environmental factors in the development of FMS is undeniable. Although several models and perspectives point in this direction (Bronfenbrenner \& Carvalho-Barreto, 2011; Clark, 2005; Gallahue \& Ozmun, 2006), few studies provide investigation of these associations (Venetsanou \& Kambas, 2009). Given the presence of FMS in most sport games, several studies used the argument of the most popular sport culture could influence and explain inter cultural differences in motor performance (Aponte, French, \& Sherrill, 1990; Chow, Henderson, \& Barnett, 2001; Miyahara et al., 1998; Wong \& Cheung, 2006). Nevertheless, to our knowledge no study investigated the direct influence of most popular sport in each country. We believe that this study furnishes an advancement in this direction.

In light of our current findings, some limitations on the study should be considered. In addition to gender differences, we must consider that studies by the USA and Hong Kong were published about nine years apart from Madeira Island. Over the years, a plethora of geographic and socioeconomic changes may have hindered the development of FMS (e.g., the decrease in the number of public spaces where children can engage in physical activities and sports). However, two multi-cohort longitudinal studies that assessed American children (age $=5$ to 13) in the same school (conducted between 1992 and 2000) showed that children who were assessed with four years apart demonstrated similar chances to performing object control skill with mastery (Butterfield et al., 2012; Loovis, Butterfield, \& Bagaka's, 2008). In this case, it seems that this period of time (9 years) could not be a factor that can explain the differences in performance between countries.
Another two limitations could be attributed to the sampling distribution and for the curriculum proposals. While Ulrich (2000) assessed American children from 10 states, other studies consider one city (Hong Kong) or region only (Madeira). Thus, our results may have been influenced by regional characteristics (e.g., regional preferences for a different sport) that in turn could restrict the development of other skills. Although the goals of the national curriculum may be considered as good indicators of the goals established in each school, their contents were not controlled in the present study.

\section{CONCLUSION}

In conclusion, the results of this study indicate that the most popular sport is not a determining factor in the level of mastery in FMS. Furthermore, we found that more direct goals in development of specific FMS in the national curriculum proposals is associated with a greater percentage of children that demonstrate mastery in these skills. In this case, considering the importance of the FMS to both development of specialized motor skills and its relation to later activities, beside the encouragement from the media, parents and friends, to increase the chances of a child becoming a physically active adult, our findings suggest the necessity of specific programs - supported by a curricular proposal with specific goals to the development of FMS, especially in the early years of school education.

\section{Acknowledgments:}

We would like to thank Thomas Novak for his corrections and comments on a previous version of this manuscript.

\section{Conflict of Interest:}

Nothing to declare 


\section{Funding}

Mateus Maia Pacheco was funded by Cnpq - protocol 211487/2013-9.

The manuscript was funded by CAPES.

\section{REFERENCES}

AAHPERD Curriculum Framework Task Force. (2014). Society of Health and Physical Educator. State University of New York College at Cortland.

Afonso, G. H., Freitas, D. H., Carmo, J. M., Lefèvre, J. A., Almeida, M. J., Lopes, V. P., ... Maia, J. (2009). [Motor performance. A normative and criterial study in children from the Autonomous Region of Madeira, Portugal]. Revista Portuguesa de Ciências do Desporto, 9(2-3), 160-174.

Aponte, R., French, R., \& Sherrill, C. (1990). Motor development of Puerto Rican children: crosscultural perspectives. Perceptual and Motor Skills, $\quad 71(3 \quad \mathrm{Pt} \quad 2), \quad 1200-1202$. http://doi.org/10.2466/pms.1990.71.3f.1200

Barnett, L. M., Van Beurden, E., Morgan, P. J., Brooks, L. O., \& Beard, J. R. (2008). Does childhood motor skill proficiency predict adolescent fitness? Medicine and Science in Sports and Exercise, 40(12),

2137-2144. http://doi.org/10.1249/MSS.0b013e31818160d 3

Barnett, L. M., van Beurden, E., Morgan, P. J., Brooks, L. O., \& Beard, J. R. (2010). Gender differences in motor skill proficiency from childhood to adolescence: a longitudinal study. Research Quarterly for Exercise and Sport, 81(2), 162-170. http://doi.org/10.1080/02701367.2010.105996 63

Beurden, E. va., Barnett, L. ., Zask, A., Dietrich, U. ., Brooks, L. ., \& Beard, J. (2003). Can we skill and activate children through primary school physical education lessons? «move it groove it»-a collaborative health promotion intervention. Preventive Medicine, 36(4), 493-501. http://doi.org/10.1016/S0091-7435(02)00044-0

Bronfenbrenner, U., \& Carvalho-Barreto, A. (2011). Bioecologia do desenvolvimento humano: tornando os seres humanos mais humanos. Porto Alegre: ARTMED.

Burton, A. W., \& Miller, D. E. (1998). Movement Skill Assessment. Champagn, IL: Human Kinetics.

Butterfield, S. A., Angell, R. M., \& Mason, C. A. (2012). Age and sex differences in object control skills by children ages 5 to 14. Perceptual and Motor Skills, 114(1), 261-274. http://doi.org/10.2466/10.11.25.PMS.114.1.261 $-274$

Chen, B. (2015). Sports in China. Obtido de http://www.foreignercn.com

Chow, S. M., Henderson, S. E., \& Barnett, A. L. (2001). The Movement Assessment Battery for Children: a comparison of 4-year-old to 6-year-old children from Hong Kong and the United States. The
American Journal of Occupational Therapy: Official Publication of the American Occupational Therapy Association, 55(1), 55-61.

Clark, J. E. (2005). From the Beginning: A Developmental Perspective on Movement and Mobility. Quest, 57(1), 37-45. http://doi.org/10.1080/00336297.2005.104918 41

Clark, J. E., \& Metcalfe, J. S. (2002). The mountain of motor development: A metaphor. Motor development: Research and reviews, 2, 163-190.

Curriculum Development Council (CDC). (2002). Physical education: Key learning area curriculum guide. Hong Kong.

DaCosta, L. P., Bitencourt, V., Nogueira, L., Miragaya, A. M., Matsudo, V., Noé, R., \& Carvalho, A. (2006). Cenário de tendências gerais dos esportes e atividades físicas no Brasil. Atlas do esporte no Brasil [Internet]. Rio de Janeiro: CONFEF, 21-3.

Departamento do Ensino Secundário. (1998). Organização Curricular e Programas: Educação Física [Organization Curriculum and Programs]. Ministério da Educação Português.

Gallahue, D. L., \& Ozmun, J. C. (2006). Understanding Motor Development: Infants, Children, Adolescents, Adults with PowerWeb. Boston: McGraw-Hill Higher Education.

Gallahue, D. L., Ozmun, J. C., \& Goodway, J. D. (2013). Understanding Motor Development: Infants, Children, Adolescents, Adults. Boston: MCGRAW-HILL Higher Education.

Goodway, J. D., Robinson, L. E., \& Crowe, H. (2010). Gender Differences in Fundamental Motor Skill Development in Disadvantaged Preschoolers From Two Geographical Regions. Research Quarterly for Exercise and Sport, 81(1), 17-24. http://doi.org/10.1080/02701367.2010.105996 24

Hardy, L. L., Barnett, L., Espinel, P., \& Okely, A. D. (2013a). Thirteen-year trends in child and adolescent fundamental movement skills: 19972010. Medicine and Science in Sports and Exercise, 45(10), 1965-1970. http://doi.org/10.1249/MSS.0b013e318295a9fc

Hardy, L. L., Barnett, L., Espinel, P., \& Okely, A. D. (2013b). Thirteen-Year Trends in Child and Adolescent Fundamental Movement Skills: 1997-2010. Medicine \& Science in Sports \& Exercise, 45(10), 1965-1970. http://doi.org/10.1249/MSS.0b013e318295a9fc

Haubenstricker, J., \& Seefeldt, V. (1986). Acquisition of motor skills during childhood. Em V. Seefeldt (Ed.), Physical activity and well-being (pp. 411021). Reston, Virginia: American Alliance for Health, Physical Education, Recreation and Dance.

Joanne Hui-Tzu Wang. (2004). A Study on Gross Motor Skills of Preschool Children. Journal of Research in Childhood Education. Obtido de http://www.tandfonline.com/doi/abs/10.1080/ 02568540409595052 
Jones, R. A., Riethmuller, A., Hesketh, K., Trezise, J., Batterham, M., \& Okely, A. D. (2011). Promoting fundamental movement skill development and physical activity in early childhood settings: a cluster randomized controlled trial. Pediatric Exercise Science, 23(4), 600-615.

Koutedakis, Y., \& Bouziotas, C. (2003). National physical education curriculum: motor and cardiovascular health related fitness in Greek adolescents. British Journal of Sports Medicine, 37(4), 311-314.

Logan, S. W., Robinson, L. E., Wilson, A. E., \& Lucas, W. A. (2012). Getting the fundamentals of movement: a meta-analysis of the effectiveness of motor skill interventions in children. Child: Care, Health and Development, 38(3), 305-315. http://doi.org/10.1111/j.13652214.2011.01307.x

Loovis, E. M., Butterfield, S. A., \& Bagaka's, J. G. (2008). Development of catching by children in kindergarten to grade 8: a multicohort longitudinal study. Perceptual and Motor Skills, 107(1), 121-128. http://doi.org/10.2466/pms.107.1.121-128

Manoel, E. J. (1994). Desenvolvimento motor: implicaçöes para a educaçäo física escolar. Revista Paulista de Educação Física, 8(1), 82-97.

Marivoet, S. (2000). Práticas desportivas na sociedade portuguesa (1988-1998). Em Actas do IV Congresso Português de Sociologia: Passados Recentes, Futuros Próximos. Obtido de http://www.aps.pt/cms/docs_prv/docs/DPR462 e088b86481 1.PDF

McKenzie, T. L., Alcaraz, J. E., Sallis, J. F., \& Faucette, F. N. (1998). Effects of a Physical Education Program on Children's Manipulative Skills. Journal of Teaching in Physical Education, 17(3), 327-341. http://doi.org/10.1123/jtpe.17.3.327

Miyahara, M., Tsujii, M., Hanai, T., Jongmans, M., Barnett, A., E. Henderson, S., ... Kageyama, H. (1998). The Movement Assessment Battery for Children: A preliminary investigation of its usefulness in Japan. Human Movement Science, 17(4-5), 679-697. http://doi.org/10.1016/S0167-9457(98)00018-9

National Association for Sport and Physical Education. (2004). Moving into the future: National standards for physical education (2. ${ }^{\mathrm{a}}$ ed.). Reston, VA: St. Louis.

Okely, A. D., \& Booth, M. L. (2004). Mastery of fundamental movement skills among children in New South Wales: prevalence and sociodemographic distribution. Journal of Science and Medicine in Sport / Sports Medicine Australia, 7(3), 358-372.

Okely, A. D., Booth, M. L., \& Patterson, J. W. (2001). Relationship of physical activity to fundamental movement skills among adolescents. Medicine and Science in Sports and Exercise, 33(11), 1899-1904.
Pang, A. W.-Y., \& Fong, D. T.-P. (2009). Fundamental motor skill proficiency of Hong Kong children aged 6-9 years. Research in Sports Medicine (Print), 17(3), 125-144. http://doi.org/10.1080/15438620902897516

Ridgway, C. L., Ong, K. K., Tammelin, T. H., Sharp, S., Ekelund, U., \& Jarvelin, M.-R. (2009). Infant motor development predicts sports participation at age 14 years: northern Finland birth cohort of 1966. Plos One, 4(8), e6837. http://doi.org/10.1371/journal.pone.0006837

Rimmer, James. H., \& Kelly, Luke. E. (1989). Gross Motor Development in Preschool Children with Learning Disabilities. Adapted Physical Activity Quarterly, pp. 268-279.

Schwartz, N., \& McGarry, T. (2014). The NFL is the most popular sport in America for the 30th year running. Obtido de http://ftw.usatoday.com/2014/01/nfl-mostpopular-sport-poll

Seefeldt, V. (1980). Developmental motor patterns: Implications for elementary school physical education. Em C. Nadeau, W. Holliwell, K. M. Holliwell, \& G. Roberts (Eds.), Psychology of motor behavior and sport (pp. 314-323). Champaign, IL: Human Kinetics.

Seefeldt, V., \& Haubenstricker, J. (1982). Patterns, phases, or stages: an analytical model for the study of developmental movement. Em J. A. S. Kelso \& J. E. Clark (Eds.), The Development of Movement Control and Coordination (pp. 309319). New York: Wiley.

Tani, G. (2011). Leituras em educação física: Retratos de uma jornada [Readings in physical education: Pictures of a journey. São Paulo: Phorte.

Thomas, J. R., \& French, K. E. (1985). Gender differences across age in motor performance a meta-analysis. Psychological Bulletin, 98(2), 260-282. http://doi.org/10.1037//00332909.98.2.260

Ulrich, D. A. (2000). Test of Gross Motor Development. Austin, Texas: Harcourt Education.

Valentini, N. C. (2012). Validity and Reliability of the TGMD-2 for Brazilian Children. Journal of Motor Behavior, 44(4), 275-280. http://doi.org/10.1080/00222895.2012.700967

van Beurden, E., Barnett, L. M., Zask, A., Dietrich, U. C., Brooks, L. O., \& Beard, J. (2003). Can we skill and activate children through primary school physical education lessons? «Move it Groove it»-a collaborative health promotion intervention. Preventive Medicine, 36(4), 493-501.

van Beurden, E., Zask, A., Barnett, L. M., \& Dietrich, U. C. (2002). Fundamental movement skills-how do primary school children perform? The "Move it Groove it» program in rural Australia. Journal of Science and Medicine in Sport / Sports Medicine Australia, 5(3), 244-252. 
Venetsanou, F., \& Kambas, A. (2009). Environmental Factors Affecting Preschoolers' Motor Development. Early Childhood Education Journal, 37(4), 319-327. http://doi.org/10.1007/s10643-009-0350-z
Wickstrom, R. L. (1977). Fundamental Motor Patterns (Vol. 1). Philadelphia: Lea \& Febiger.

Wong, A. K. Y., \& Cheung, S. Y. (2006). Gross motor skills performance of Hong Kong Chinese children. Journal of Physical Education \& Recreation, 12(2), 23-29. 